\title{
COMMISSIONS MIXTES
}

(Membres additionnels de Commissions mixtes élus par l'Union

Internationale de Géodésie et Géophysique.)

18. Commission des Longitudes par Télegraphie sans Fil.

Membres: MM. W. Bowie, Hinks, Jelstrup, Jolly, Spencer Jones, Jouaust, Ogilvie, Somville.

19. Commission de la Variation des Latitudes.

Membres: MM. Bowie, Brillouin, F. M. da Costa Lobo, Jelstrup, A. Lambert, Nušl, Rosén, Stroobant.

31. Commission de l'Heure.

Membres: MM. Baeschlin, Boulnois, Bowie, F. M. da Costa Lobo, de Lemos, Dodwell, Ferri, Forni, Gastardi, Giotti, S. Hirayama, Hunter, Jelstrup, Jolly, Kennelly, Mailles, Nijland, Nušl, Ogilvie, Romagna-Manoia, Sánchez, Seligmann, Tanakadate, Warchalowski. 